\title{
Consumo de carnes por adolescentes do Sul do Brasil
}

\author{
Meat consumption among Southern \\ Brazilian adolescents
}

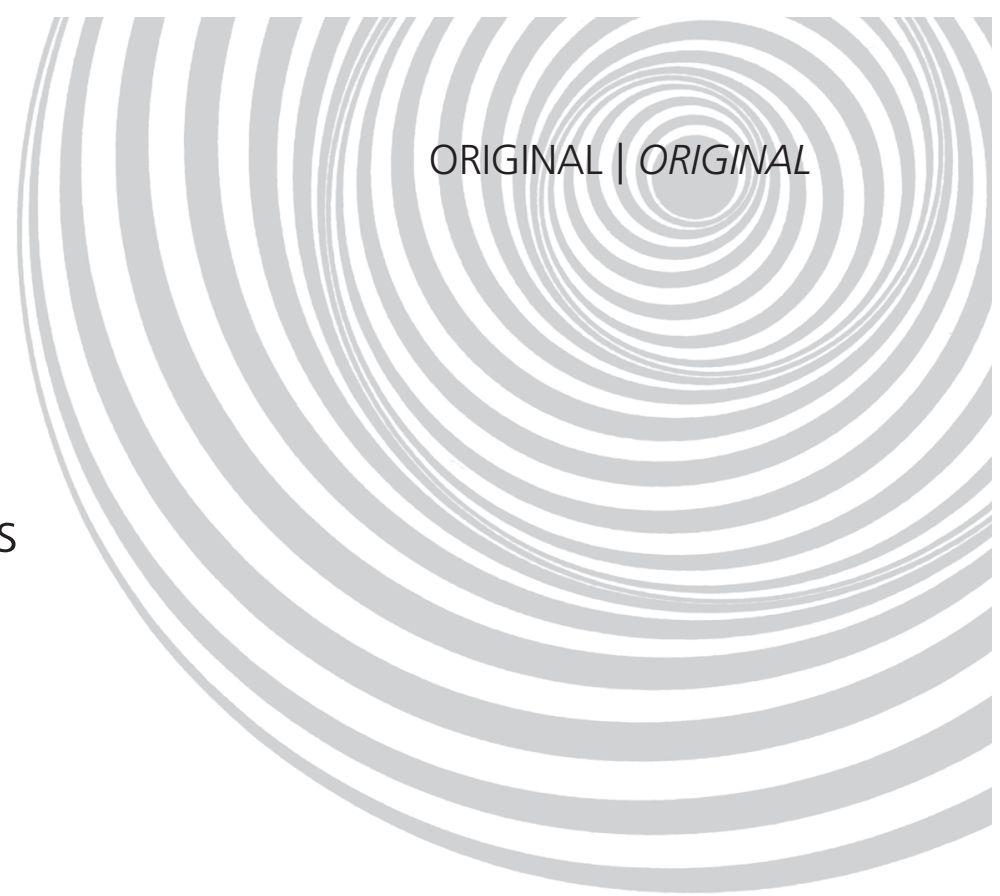

Maria Cecília Formoso ASSUNÇÃO ${ }^{1}$

Samuel Carvalho DUMITH ${ }^{1}$

Ana Maria Baptista MENEZES ${ }^{1}$

Cora Luíza ARAÚJO'

Bruna Celestino SCHNEIDER ${ }^{1}$

Carolina Ávila VIANNA ${ }^{1}$

Eduardo Coelho MACHADO'

Fernando César WEHRMEISTER ${ }^{1}$

Ludmila Correa MUNIZ

Roberta de Vargas ZANINI ${ }^{1}$

Silvana Paiva ORLANDI ${ }^{1}$

Samanta Winck MADRUGA'

\section{RE S U M O}

\section{Objetivo}

Descrever a frequência de consumo de diferentes tipos de carnes conforme variáveis sociodemográficas e nutricionais, e analisar o consumo, em adolescentes do Sul do Brasil, de dieta rica em gordura conforme os tipos de carne consumidos.

\section{Métodos}

Estudo transversal realizado com adolescentes pertencentes à coorte de nascimentos de 1993, em Pelotas (RS). A frequência de consumo de carnes vermelhas, brancas, vísceras e embutidos foi avaliada por um Questionário de Frequência de Consumo Alimentar, adaptado para este estudo. A ingestão de dieta rica em gordura foi avaliada a partir do instrumento proposto por Block. As variáveis independentes foram sexo, cor da pele, nível socioeconômico, escolaridade materna e estado nutricional. Nas análises estatísticas, foram utilizados testes Qui-quadrado de heterogeneidade e de tendência linear.

\section{Resultados}

Foram avaliados 4325 adolescentes com idade média de 14,7, DP=0,3 anos, dos quais 51,2\% eram do sexo feminino. A frequência de consumo diário de carnes vermelhas foi maior do que a de carnes brancas $(43,0 \%$

\footnotetext{
${ }^{1}$ Universidade Federal de Pelotas, Programa de Pós-Graduação em Epidemiologia. R. Marechal Deodoro, 1160, $3^{\circ}$ piso, 96020-220, Pelotas, RS, Brasil. Correspondência para/Correspondence to: SC DUMITH. E-mail: <scdumith@yahoo.com.br>.
} 
464 | MCF ASSUNÇÃO et al.

e $9,7 \%$, respectivamente). O consumo de embutidos por mais do que quatro vezes por semana foi referido por $48,5 \%$ dos indivíduos, e $81,4 \%$ relataram consumir vísceras raramente ou nunca. Adolescentes de maior nível socioeconômico e filhos de mães com maior escolaridade apresentaram maior consumo de carnes vermelhas e embutidos, enquanto aqueles em situação oposta apresentaram maior consumo de carnes brancas. Adolescentes que consomem carnes com maior frequência também consomem dieta rica em gordura.

\section{Conclusão}

As carnes consumidas com maior frequência pelos adolescentes foram as vermelhas e os embutidos. No entanto, adolescentes de maior nível socioeconômico consomem carnes vermelhas com maior frequência, enquanto adolescentes menos favorecidos economicamente consomem mais carnes brancas.

Termos de indexação: Adolescentes. Carne. Consumo de alimentos.

\section{A B S T R A C T}

\section{Objective}

The present study investigated the consumption frequency of various meats according to sociodemographic and nutritional variables and analyzed the consumption of high-fat diets according to type of meat by southern Brazilian adolescents.

\section{Methods}

This cross-sectional study included the 1993 birth cohort from Pelotas (RS). The consumption frequency of red, white and organ meats and sausage were investigated by a food frequency questionnaire adapted for this study. Dietary fat content was determined as proposed by Block. The independent variables were gender, skin color, socioeconomic level, maternal education level and nutritional status. The statistical analyses included the Chi-square linear tendency test and the Chi-square test of homogeneity.

\section{Results}

A total of 4325 adolescents with a mean age of 14.7 years, SD=0.3 years, were assessed, of which $51.2 \%$ were females. Red meat was consumed more frequently daily than white meat $(43.0 \%$ and $9.7 \%$, respectively). Sausage consumption frequency greater than 4 times per week was reported by $48.5 \%$ of the sample, and $81.4 \%$ reported to consume organ meats rarely or never. Adolescents of higher socioeconomic levels and of mothers with higher education levels consumed more red meats and sausages, while those in the opposite situation consumed more white meats. Adolescents who consumed meats more frequently also consumed a high-fat diet.

\section{Conclusion}

Red meats and sausages were the meats most frequently consumed by the study adolescents. However, adolescents of higher socioeconomic levels consumed red meats more often, while those of lower socioeconomic levels consumed white meats more often.

Indexing terms: Adolescents. Meat. Food consumption.

\section{N T R O D U ÇÃ O}

Os seres humanos são onívoros, isto é, alimentam-se de uma enorme variedade de alimentos tanto de origem animal como vegetal. Desde a pré-história, a carne e outros alimentos de origem animal fazem parte da dieta humana. Alimentos de origem animal incluem carnes, vísceras, aves, peixes, ovos e leite e derivados. No Brasil, as carnes mais consumidas são as bovinas, as suínas, as de aves e as de peixes ${ }^{1}$.
As carnes e os peixes, em geral, são fontes importantes de proteínas de elevado valor biológico, bem como de ferro e de outros nutrientes com propriedades funcionais ${ }^{1}$. As vísceras, assim como as carnes e peixes, também são boas fontes de ferro, entretanto apresentam alto teor de gordura saturada e colesterol' . Os produtos derivados de carnes, como salsichas e embutidos, têm quantidades bastante elevadas de gorduras e alto teor de sal, e devem, portanto, ser evitados. Esses alimentos são considerados nutritivos devido aos 
seus aspectos nutricionais desde que consumidos com moderação. O consumo elevado de gorduras, especialmente de origem animal, aumentam o risco para o desenvolvimento de doenças cardiovasculares, assim como para alguns tipos de câncer-2-4. Estudos recentes têm demonstrado relação direta entre consumo de carne e ingestão elevada de gorduras, devido ao alto conteúdo de colesterol e gordura saturada do alimento². Dessa forma, o consumo inadequado de carnes, especialmente das vermelhas e dos embutidos, vem sendo apontado como um dos principais fatores de risco para o aumento da carga global de doenças em todo o mundo². Diante disso, o Ministério da Saúde, por meio do Guia Alimentar para a População Brasileira, recomenda o consumo diário de uma porção de carne ou peixes, ambos com baixo teor de gordura'.

Considerando que o padrão alimentar da população está diretamente ligado à morbimortalidade por doenças crônicas ao longo de todas as fases da vida², e que hábitos alimentares adquiridos na adolescência podem permanecer na idade adulta, repercutindo, muitas vezes, de maneira negativa sobre a saúde desses indivíduos ${ }^{5,6}$, o presente estudo teve como objetivo descrever a frequência de consumo de diferentes tipos de carnes conforme variáveis sociodemográficas e nutricionais entre os adolescentes de 15 anos de idade pertencentes à coorte de nascimentos de 1993 em Pelotas (RS). Além disso, avaliou-se o consumo de dieta rica em gordura conforme os tipos de carne consumidos por esses adolescentes.

\section{MÉ T O D O S}

Os dados do presente estudo são referentes ao acompanhamento realizado de janeiro a agosto de 2008 com os indivíduos pertencentes à coorte dos nascidos em 1993, na cidade de Pelotas (RS). Dos 5249 nascidos-vivos no ano de 1993, foram localizados 4349 (85,7\% da coorte original), e, destes, 4325 foram efetivamente entrevistados.
As informações sobre "Frequência de consumo de carnes" foram obtidas a partir de um Questionário de Frequência de Consumo Alimen$\operatorname{tar}$ (QFCA) adaptado de Sichieri \& Everhart? ${ }^{7}$, validado a partir de dados de uma comunidade universitária do Rio de Janeiro. O questionário, composto por 81 itens alimentares, apresenta a frequência de consumo coletada de forma aberta (número de vezes de zero a dez e opção de frequência de consumo diário, semanal, mensal ou anual). Os tipos de carne foram agrupados em: carne vermelha (com osso, tipo costela, paleta, agulha etc; sem osso, tipo bife, carne assada, guisado e carne de porco), carne branca (frango e peixe: peixe fresco, camarão e peixe enlatado) e embutidos (presunto ou mortadela, salsicha ou linguiça, e alimentos cárneos conservados em sal, como charque). A frequência de consumo de vísceras (rim, fígado, coração, moela e mondongo) também foi avaliada. Optou-se por categorizar a frequência de consumo dos grupos de carnes em raramente ou nunca (menos de uma vez por semana), de um a três vezes por semana, de quatro a seis vezes por semana e diariamente.

Uma segunda análise avaliou a relação entre o consumo de carnes e o consumo de uma dieta rica em gorduras. Para avaliar o teor de gordura da dieta, foi utilizado o questionário proposto por Block $^{8}$, dividido em duas partes. A primeira, composta de 15 itens alimentares, visa identificar a frequência de consumo de alimentos ricos em gordura. A segunda parte, composta de nove itens, avalia a ingestão de alimentos ricos em fibras. No presente estudo, utilizou-se apenas o bloco referente ao consumo de gorduras. Esse questionário apresenta um autoescore, e foi considerado como tendo uma dieta rica em gordura o adolescente que obteve escore superior a 27 pontos.

As variáveis independentes utilizadas foram sexo, cor da pele (autorreferida pelo adolescente), nível socioeconômico (classificado conforme o índice de bens e dividido em quintis, classificados em ordem crescente: o $1^{\circ}$ quintil é o grupo menos favorecido economicamente), escolaridade 
da mãe (anos completos de estudo) e estado nutricional (classificado a partir do Índice de Massa Corporal - IMC, segundo idade e sexo, de acordo com os critérios propostos pela Organização Mundial de Saúde) ${ }^{9}$ e a frequência de consumo de carnes, obtida pelo QFCA.

Os adolescentes foram pesados com auxílio de balanças digitais da marca Tanita ${ }^{\circledR}$, com capacidade máxima de $150 \mathrm{~kg}$ e precisão de $100 \mathrm{~g}$, e medidos com estadiômetros de alumínio. Esse procedimento foi realizado por antropometristas treinadas e submetidas a processo de padronização para a coleta das medidas.

A coleta dos dados se deu por entrevistas domiciliares efetuadas por entrevistadoras previamente treinadas, com ensino médio completo e maiores de 18 anos. O controle de qualidade das entrevistas foi realizado por revisita a $10 \%$ dos entrevistados e a partir de contato telefônico a outros 20\% para aplicação de uma versão reduzida do questionário. Maiores detalhes metodológicos sobre o estudo estão descritos em outra publicação ${ }^{10}$.

Os dados foram duplamente digitados no programa Epi Info 6.0 e para as análises estatísticas foi utilizado o pacote estatístico Stata 11.0. A análise dos dados incluiu a descrição da amostra e, para a análise bivariada, foi utilizado o teste Qui-quadrado (de heterogeneidade ou tendência linear) a fim de detectar possíveis diferenças entre as variáveis analisadas. Para a obtenção dos valores $p$, foram criadas variáveis dummy binárias apenas para as categorias extremas do consumo de cada tipo de carne (exemplo: consome diariamente versus não consome diariamente. Para avaliação da associação entre consumo de dieta rica em gordura e consumo de carnes foi utilizado o teste Qui-quadrado de tendência linear.

O projeto foi submetido e aprovado pelo Comitê de Ética em Pesquisa da Faculdade de Medicina da Universidade Federal de Pelotas, ofício 158/07. O Termo de Consentimento Livre e Esclarecido foi assinado pelos adolescentes e pelos seus pais/responsáveis.
RESULTA DOS

A análise dos 4325 adolescentes avaliados identificou que a idade média foi de 14,7 anos (Desvio-Padrão - DP=0,3). Houve um discreto predomínio do sexo feminino (51,2\%), 64,0\% dos indivíduos eram de cor de pele branca, 41,7\% consumiam dieta rica em gordura e $27,6 \%$ dos adolescentes foram classificados com algum grau de excesso de peso.

Carnes vermelhas foram consumidas diariamente por $43,0 \%$ da amostra, enquanto apenas 4,0\% referiram não comer ou consumir raramente o alimento. Em relação aos embutidos, o segundo grupo de carnes mais frequentemente consumido: $28,3 \%$ dos adolescentes referiram ingestão diária e $87,4 \%$ relataram um consumo semanal. As vísceras, por outro lado, foram muito pouco consumidas pelos adolescentes, visto que $81,4 \%$ deles relataram consumi-las raramente ou nunca. Entretanto, 17,3\% referiram o consumo de vísceras entre uma e três vezes por semana. Em relação ao grupo das carnes brancas, 85,4\% dos adolescentes referiram ter o hábito de consumir esse tipo de carne pelo menos uma vez por semana. Entre os tipos de carnes brancas, cabe ressaltar que os peixes apresentaram menor frequência de consumo (72,4\% relataram consumir raramente ou nunca), enquanto $54,4 \%$ consumiam frango pelo menos uma vez por semana. A prevalência de adolescentes que referiram não ter o hábito de consumir qualquer tipo de carne foi de $1,5 \%$ (Figura 1).

Observou-se que o consumo diário de carnes vermelhas foi discretamente superior entre adolescentes do sexo feminino, de cor da pele branca, de maior nível socioeconômico e entre aqueles cujas mães tinham mais de 11 anos de escolaridade (Tabela 1).

Em relação ao hábito de consumir carnes brancas, a maior frequência de consumo diário foi observada entre os adolescentes de menor nível socioeconômico $(14,4 \%)$ e naqueles filhos de mães de menor escolaridade (14,7\%). A menor frequência de consumo diário de carnes brancas 
Tabela 1. Frequência de consumo de carnes vermelhas segundo características sociodemográficas e nutricionais ( $n=4$ 235). Pelotas (RS), 2008.

\begin{tabular}{|c|c|c|c|c|}
\hline \multirow{2}{*}{ Variáveis } & \multicolumn{4}{|c|}{ Frequência de consumo - \% (IC95\%) } \\
\hline & $<1$ x/semana & $1-3 x /$ semana & 4-6 x/semana & Diariamente \\
\hline Sexo & $p=0,04^{*}$ & & & $p=0,1^{*}$ \\
\hline Masculino & $3,4(2,6 ; 4,1)$ & $23,2(21,4 ; 25,0)$ & $31,7(29,7 ; 33,7)$ & $41,8(31,7 ; 43,9)$ \\
\hline Feminino & $4,6(3,7 ; 5,4)$ & $20,2(18,6 ; 21,9)$ & $31,0(29,1 ; 33,0)$ & $44,2(42,1 ; 46,2)$ \\
\hline Cor da pele & $p=0,2^{*}$ & & & $p=0,1^{*}$ \\
\hline Branca & $3,7(3,0 ; 4,4)$ & $20,1(18,6 ; 21,6)$ & $32,4(30,6 ; 34,1)$ & $43,9(42,0 ; 45,7)$ \\
\hline Preta/Parda & $4,3(3,2 ; 5,4)$ & $24,2(21,9 ; 26,4)$ & $29,4(27,0 ; 31,8)$ & $42,2(39,6 ; 44,7)$ \\
\hline Outros & $6,3(2,5 ; 10,1)$ & $26,4(19,5 ; 33,3)$ & $31,4(24,2 ; 38,7)$ & $35,8(28,4 ; 43,3)$ \\
\hline Nível socioeconômico (Índice de bens) & $p<0,001^{* *}$ & & & $p<0,001^{* *}$ \\
\hline $1^{\circ}$ quintil (menor) & $6,9(5,2 ; 8,6)$ & $26,4(23,5 ; 29,4)$ & $27,9(25,0 ; 30,9)$ & $38,7(35,4 ; 41,9)$ \\
\hline $2^{\circ}$ & $4,6(3,2 ; 6,0)$ & $23,1(20,3 ; 26,0)$ & $32,9(29,8 ; 36,1)$ & $39,4(36,1 ; 42,6)$ \\
\hline $3^{\circ}$ & $2,4(1,4 ; 3,5)$ & $22,1(19,3 ; 24,9)$ & $31,5(28,4 ; 34,6)$ & $44,0(40,6 ; 47,3)$ \\
\hline $4^{\circ}$ & $2,7(1,6 ; 3,7)$ & $18,0(15,5 ; 20,6)$ & $31,7(28,6 ; 34,8)$ & $47,6(44,3 ; 51,0)$ \\
\hline $5^{\circ}$ quintil (maior) & $3,4(2,2 ; 4,6)$ & $18,2(15,6 ; 20,8)$ & $33,0(29,9 ; 36,2)$ & $45,4(42,1 ; 48,7)$ \\
\hline Escolaridade materna (anos de estudo) & $p<0,001^{* *}$ & & & $p=0,6^{* \star}$ \\
\hline $0-4$ & $5,8(4,2 ; 7,4)$ & $24,5(21,7 ; 27,2)$ & $27,3(24,4 ; 30,1)$ & $42,4(39,2 ; 45,6)$ \\
\hline $5-8$ & $4,4(3,4 ; 5,4)$ & $21,5(19,6 ; 23,5)$ & $32,0(29,7 ; 34,2)$ & $42,1(39,7 ; 44,5)$ \\
\hline $9-11$ & $2,7(1,7 ; 3,8)$ & $21,1(18,5 ; 23,7)$ & $34,1(31,1 ; 37,2)$ & $42,0(38,8 ; 45,1)$ \\
\hline$>11$ & $2,0(0,8 ; 3,3)$ & $18,2(14,8 ; 21,7)$ & $35,0(30,8 ; 39,3)$ & $44,7(40,3 ; 49,1)$ \\
\hline Estado nutricional & $p=0,9^{* *}$ & & & $p=0,6^{* \star}$ \\
\hline Magreza & $1,6(0,0 ; 4,9)$ & $26,2(15,1 ; 37,4)$ & $37,7(25,4 ; 50,0)$ & $34,4(22,4 ; 46,5)$ \\
\hline Eutrofia & $3,8(3,1 ; 4,5)$ & $22,3(20,8 ; 23,8)$ & $31,4(29,7 ; 33,1)$ & $42,5(40,7 ; 44,3)$ \\
\hline Sobrepeso & $4,9(3,4 ; 6,4)$ & $19,5(16,7 ; 22,2)$ & $30,5(27,3 ; 33,8)$ & $45,1(41,6 ; 48,6)$ \\
\hline Obesidade & $2,8(1,1 ; 4,5)$ & $22,8(18,4 ; 27,2)$ & $33,2(28,3 ; 38,1)$ & $41,1(36,0 ; 46,3)$ \\
\hline
\end{tabular}

*Teste Qui-quadrado de heterogeneidade; ${ }^{* *}$ Teste Qui-quadrado de tendência linear; IC: Intervalo de Confiança.

foi evidenciada entre os indivíduos de cor da pele branca (Tabela 2).

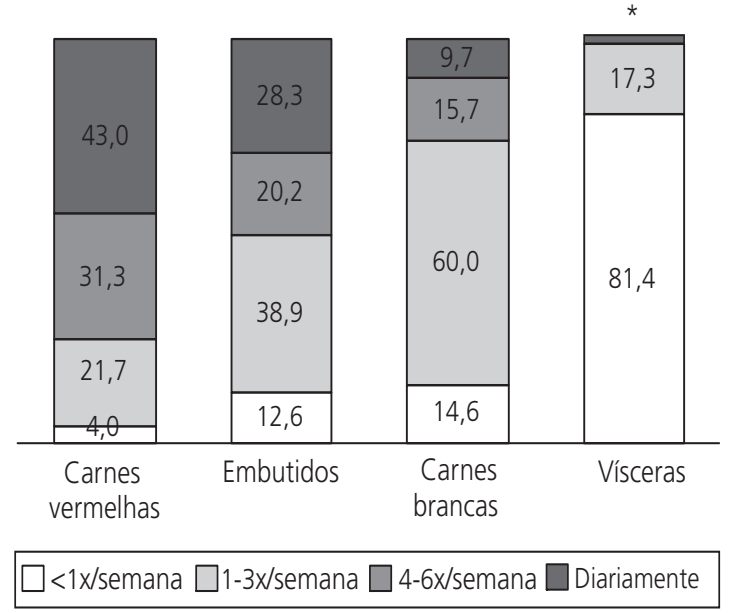

Figura 1. Frequência (\%) do consumo de diferentes tipos de carnes entre adolescentes ( $n=4325)$. Pelotas (RS), 2008.

Nota: * Frequência 4-6 x/semana $=0,8 \%$; Frequência diária $=0,5 \%$.
Observa-se que cerca de $40 \%$ dos adolescentes consomem alimentos embutidos entre uma e três vezes por semana. A maior frequência de consumo de embutidos foi evidenciada entre os adolescentes de maior nível socioeconômico e naqueles filhos de mães com maior escolaridade (Tabela 3).

Para os três tipos de carnes avaliados (vísceras não foi incluído devido ao baixo consumo), observou-se relação direta entre maior frequência de consumo e dieta rica em gordura. Consumidores diários de carnes vermelhas apresentaram uma frequência de dieta rica em gordura 2,5 vezes maior do que aqueles com consumo inferior a uma vez por semana. Na comparação dos mesmos grupos de frequência de consumo para os outros tipos de carnes, encontrou-se que o consumo diário de embutidos elevou o risco para o 
468 | MCF ASSUNÇÃO et al.

Tabela 2. Frequência de consumo de carnes brancas segundo características sociodemográficas e nutricionais ( $n=4$ 235). Pelotas (RS), 2008.

\begin{tabular}{|c|c|c|c|c|}
\hline \multirow{2}{*}{ Variáveis } & \multicolumn{4}{|c|}{ Frequência de consumo - \% (IC95\%) } \\
\hline & $<1 \mathrm{x} / \mathrm{semana}$ & 1-3 x/semana & 4-6 x/semana & Diariamente \\
\hline Sexo & $p=0,06^{*}$ & & & $p=0,3^{*}$ \\
\hline Masculino & $15,6(14,1 ; 17,2)$ & $58,0(55,9 ; 60,1)$ & $16,2(14,6 ; 17,8)$ & $10,2(8,9 ; 11,5)$ \\
\hline Feminino & $13,6(12,2 ; 15,0)$ & $61,8(59,8 ; 63,8)$ & $15,3(13,8 ; 16,8)$ & $9,3(8,1 ; 10,5)$ \\
\hline Cor da pele & $p=0,4^{*}$ & & & $p<0,001^{*}$ \\
\hline Branca & $14,4(13,1 ; 15,7)$ & $62,4(60,6 ; 64,2)$ & $15,1(13,7 ; 16,4)$ & $8,2(7,1 ; 9,2)$ \\
\hline Preta/Parda & $14,6(12,8 ; 16,5)$ & $55,9(53,3 ; 58,5)$ & $16,6(14,7 ; 18,6)$ & $12,8(11,1 ; 14,6)$ \\
\hline Outros & $18,2(12,1 ; 24,3)$ & $52,2(44,4 ; 60,0)$ & $20,1(13,9 ; 26,4)$ & $9,4(4,9 ; 14,0)$ \\
\hline Nível socioeconômico (Índice de bens) & $p=0,003^{* *}$ & & & $p<0,001^{* *}$ \\
\hline $1^{\circ}$ quintil (menor) & $17,2(14,7 ; 19,7)$ & $51,0(47,7 ; 54,4)$ & $17,3(14,8 ; 19,8)$ & $14,4(12,1 ; 16,8)$ \\
\hline $2^{\circ}$ & $15,7(13,2 ; 18,1)$ & $55,7(52,4 ; 59,1)$ & $16,8(14,3 ; 19,3)$ & $11,8(9,6 ; 14,0)$ \\
\hline $3^{\circ}$ & $13,8(11,5 ; 16,1)$ & $62,2(59,0 ; 65,5)$ & $15,3(12,9 ; 17,8)$ & $8,6(6,7 ; 10,5)$ \\
\hline $4^{\circ}$ & $13,5(11,2 ; 15,8)$ & $63,5(60,3 ; 66,7)$ & $14,5(12,1 ; 16,8)$ & $8,6(6,7 ; 10,4)$ \\
\hline $5^{\circ}$ quintil (maior) & $12,7(10,5 ; 15,0)$ & $67,2(64,1 ; 70,4)$ & $14,7(12,3 ; 17,1)$ & $5,4(3,9 ; 6,9)$ \\
\hline Escolaridade materna (anos de estudo) & $p=0,07^{* *}$ & & & $p<0,001^{* *}$ \\
\hline $0-4$ & $15,6(13,2 ; 17,9)$ & $52,8(49,6 ; 56,0)$ & $16,9(14,5 ; 19,3)$ & $14,7(12,4 ; 17,0)$ \\
\hline $5-8$ & $15,5(13,8 ; 17,2)$ & $57,6(55,2 ; 60,0)$ & $17,0(15,2 ; 18,8)$ & $9,9(8,5 ; 11,3)$ \\
\hline 9-11 & $14,1(11,8 ; 16,3)$ & $65,9(62,8 ; 68,9)$ & $14,2(11,9 ; 16,4)$ & $5,9(4,4 ; 7,4)$ \\
\hline$>11$ & $12,3(9,4 ; 15,2)$ & $68,9(64,7 ; 73,0)$ & $13,5(10,5 ; 16,6)$ & $5,3(3,3 ; 7,3)$ \\
\hline Estado nutricional & $p=0,03^{* *}$ & & & $p=0,8^{* *}$ \\
\hline Magreza & $18,0(8,3 ; 27,8)$ & $57,4(44,9 ; 69,9)$ & $9,8(2,3 ; 17,4)$ & $14,8(5,8 ; 23,7)$ \\
\hline Eutrofia & $15,3(14,0 ; 16,6)$ & $59,7(57,9 ; 61,5)$ & $15,5(14,2 ; 16,8)$ & $9,5(8,5 ; 10,6)$ \\
\hline Sobrepeso & $12,6(10,3 ; 15,0)$ & $62,5(59,1 ; 65,9)$ & $15,2(12,7 ; 17,7)$ & $9,7(7,6 ; 11,7)$ \\
\hline Obesidade & $12,4(9,0 ; 15,8)$ & $58,9(53,7 ; 64,0)$ & $18,0(14,0 ; 22,0)$ & $10,7(7,5 ; 13,9)$ \\
\hline
\end{tabular}

* Teste Qui-quadrado de heterogeneidade; ** Teste Qui-quadrado de tendência linear; IC: Intervalo de Confiança.

consumo de uma dieta rica em gordura em cerca de 3 vezes e o de carnes brancas em cerca de 1,5 vezes (Figura 2).

\section{DIS CUSS Ã O}

O presente estudo é relevante uma vez que a bibliografia em relação ao consumo de carnes entre adolescentes é escassa, e também pelo fato de a Região Sul do Brasil ser culturalmente reconhecida por esse hábito. Dentre as limitações do estudo está o fato de a análise transversal abranger somente o grupo de idade na faixa etária da adolescência: os dados analisados referem-se apenas ao acompanhamento de indivíduos de 14 e 15 anos da coorte de nascidos vivos em Pelotas (RG) no ano de 1993. Deve-se mencionar, ainda, que o instrumento utilizado, por ser um ques- tionário de frequência de consumo sem o componente quantitativo, impossibilitou conhecer as porções dos alimentos consumidos pelos adolescentes, inviabilizando, assim, a análise de adequação de consumo em relação à recomendação dada pelo Ministério da Saúde do Brasil. Além disso, o instrumento utilizado para avaliar o consumo de gorduras não foi validado especificamente para adolescentes, entretanto outros estudos com a mesma população utilizaram esse instrumento, apresentando resultados satisfatórios ${ }^{11,12}$.

Os resultados mostraram uma prevalência de consumo diário de carnes vermelhas de 43,0\% entre os adolescentes avaliados. Resultado semelhante foi observado na Pesquisa de Orçamentos Familiares (POF) 2008-2009³, que mostrou que $48,7 \%$ dos indivíduos com 10 anos ou 
Tabela 3. Frequência de consumo de embutidos segundo características sociodemográficas e nutricionais (n=4 235). Pelotas (RS), 2008.

\begin{tabular}{|c|c|c|c|c|}
\hline \multirow{2}{*}{ Variáveis } & \multicolumn{4}{|c|}{ Frequência de consumo - \% (IC95\%) } \\
\hline & $<1$ x/semana & $1-3 x /$ semana & 4-6 x/semana & Diariamente \\
\hline Sexo & $p=0,009^{*}$ & & & $p=0,2^{*}$ \\
\hline Masculino & $11,3(9,9 ; 12,6)$ & $38,0(35,9 ; 40,1)$ & $21,4(19,6 ; 23,1)$ & $29,4(27,4 ; 31,3)$ \\
\hline Feminino & $13,9(12,5 ; 15,4)$ & $39,7(37,7 ; 41,8)$ & $19,1(17,5 ; 20,7)$ & $27,2(25,4 ; 29,1)$ \\
\hline Cor da pele & $p=0,2^{*}$ & & & $p=0,1^{*}$ \\
\hline Branca & $12,0(10,8 ; 13,2)$ & $38,8(37,0 ; 40,6)$ & $20,4(18,9 ; 21,9)$ & $28,8(27,1 ; 30,5)$ \\
\hline Preta/Parda & $13,5(11,8 ; 15,3)$ & $39,2(36,4 ; 41,8)$ & $19,2(17,1 ; 21,3)$ & $28,0(25,7 ; 30,4)$ \\
\hline Outros & $15,1(9,5 ; 20,7)$ & $37,1(29,6 ; 44,6)$ & $26,4(19,5 ; 33,3)$ & $21,4(15,0 ; 27,8)$ \\
\hline Nível socioeconômico (Índice de bens) & $p=0,003^{* *}$ & & & $p<0,001^{* *}$ \\
\hline $1^{\circ}$ quintil (menor) & $16,9(14,4 ; 19,4)$ & $40,5(37,3 ; 43,8)$ & $17,0(14,5 ; 19,5)$ & $25,6(22,7 ; 28,5)$ \\
\hline $2^{\circ}$ & $11,8(9,6 ; 14,0)$ & $41,5(38,2 ; 44,8)$ & $21,5(18,7 ; 24,2)$ & $25,2(22,3 ; 28,1)$ \\
\hline $3^{\circ}$ & $11,7(9,6 ; 13,9)$ & $40,9(37,6 ; 44,2)$ & $20,1(17,4 ; 22,8)$ & $27,2(24,2 ; 30,2)$ \\
\hline $4^{\circ}$ & $11,0(8,9 ; 13,1)$ & $38,5(35,3 ; 41,7)$ & $20,6(17,9 ; 23,3)$ & $29,9(26,9 ; 33,0)$ \\
\hline $5^{\circ}$ quintil (maior) & $11,9(9,7 ; 14,1)$ & $32,7(29,5 ; 35,8)$ & $21,9(19,2 ; 24,7)$ & $33,5(30,3 ; 36,7)$ \\
\hline Escolaridade materna (anos de estudo) & $p=0,04^{* *}$ & & & $p=0,03^{* *}$ \\
\hline $0-4$ & $16,1(13,8 ; 18,5)$ & $40,6(37,4 ; 43,8)$ & $17,0(14,6 ; 19,4)$ & $26,3(23,5 ; 29,1)$ \\
\hline $5-8$ & $11,3(9,8 ; 12,9)$ & $40,6(38,2 ; 43,0)$ & $21,2(19,2 ; 23,1)$ & $26,9(24,8 ; 29,3)$ \\
\hline 9-11 & $11,3(9,3 ; 13,3)$ & $35,4(32,4 ; 38,5)$ & $22,9(20,3 ; 25,6)$ & $30,3(27,4 ; 33,3)$ \\
\hline$>11$ & $13,1(10,1 ; 16,1)$ & $36,7(32,4 ; 41,0)$ & $20,1(16,5 ; 23,6)$ & $30,1(26,0 ; 34,2)$ \\
\hline Estado nutricional & $p=0,04^{* *}$ & & & $p=0,02^{* *}$ \\
\hline Magreza & $13,1(4,6 ; 21,7)$ & $44,3(31,7 ; 56,8)$ & $19,7(9,6 ; 29,7)$ & $23,0(12,3 ; 33,6)$ \\
\hline Eutrofia & $13,1(11,8 ; 14,3)$ & $38,9(37,1 ; 40,6)$ & $20,2(18,7 ; 21,6)$ & $27,9(26,3 ; 29,5)$ \\
\hline Sobrepeso & $12,1(9,8 ; 14,4)$ & $39,6(36,1 ; 43,0)$ & $20,6(17,8 ; 23,5)$ & $27,7(24,6 ; 30,9)$ \\
\hline Obesidade & $9,0(6,0 ; 12,0)$ & $36,3(31,3 ; 41,3)$ & $19,4(15,3 ; 23,6)$ & $35,2(30,2 ; 40,2)$ \\
\hline
\end{tabular}

* Teste Qui-quadrado de heterogeneidade; ${ }^{* *}$ Teste Qui-quadrado de tendência linear; IC: Intervalo de Confiança.

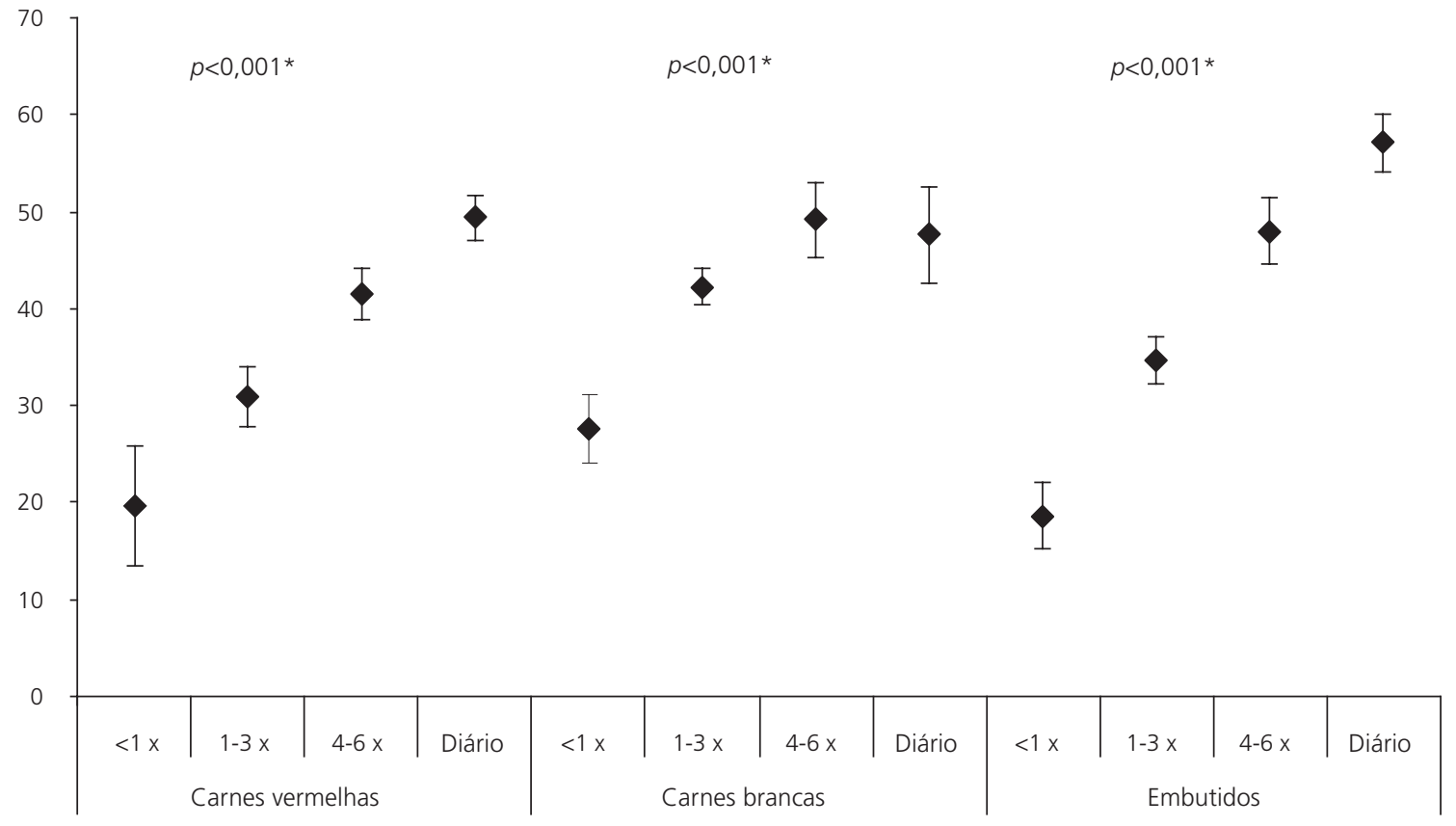

Figura 2. Prevalência de dieta rica em gorduras conforme a frequência de consumo de diferentes tipos de carnes entre adolescentes $(n=4$ 325). Pelotas (RS), 2008. 
mais residentes nas cinco regiões brasileiras consumiam carne bovina diariamente, destacando-se a Região Centro-Oeste $(88,1 \%)$. No mesmo sentido, um estudo realizado no Paraná, com adolescentes de 14 a 19 anos, mostrou que $40,3 \%$ referiram consumir diariamente esse tipo de carne ${ }^{14}$. Entretanto, o consumo diário de carne vermelha, relatado por adolescentes mexicanos de 12 a 19 anos, foi de apenas 12,5\%, valor bastante inferior ao observado no presente estudo ${ }^{15}$. A maior prevalência de consumo diário de carne vermelha observada no Paraná, nas diferentes regiões brasileiras e no presente estudo, pode, de certa forma, ser explicada pelo hábito cultural de consumir carnes vermelhas nessas regiões e também pela questão econômica, uma vez que esse alimento onera as despesas com alimentação. Mas, apesar de a carne vermelha ser um importante alimento da dieta, seu consumo excessivo, principalmente daquelas com alto teor de gordura, acarreta prejuízos para a saúde da população. Baseado nisso, o Ministério da Saúde (MS) recomenda o consumo diário de uma porção de carne magra e sem gordura aparente ${ }^{1}$.

Além disso, o MS, através do Guia Alimentar para a População Brasileira, recomenda o consumo ocasional de alimentos embutidos ${ }^{1}$. Entretanto, o consumo em uma frequência maior de quatro vezes por semana foi referido por cerca de $50,0 \%$ dos adolescentes avaliados, resultado superior ao encontrado em um estudo realizado no Ceará, onde a prevalência foi de $22,4 \%{ }^{16}$. Tal achado é preocupante visto que metade dos adolescentes avaliados referiu consumir com elevada frequência embutidos, alimentos de baixo valor nutricional, extremamente ricos em gorduras saturadas e sal, e relacionados a um maior risco para o desenvolvimento de doenças crônicas não transmissíveis.

Em relação ao consumo de vísceras, observou-se que mais de $80 \%$ dos adolescentes relataram consumi-las raramente ou nunca, não atingindo, dessa forma, a recomendação preconizada pelo MS de ingerir pelo menos uma vez por semana vísceras e miúdos, como fígado bovino, coração de galinha entre outros ${ }^{1}$. No mesmo sentido, Dalla-Costa et al. ${ }^{14}$ realizaram um estudo no município de Toledo (PR) e encontraram que aproximadamente $70 \%$ dos adolescentes avaliados não consumiam esse tipo de carne ${ }^{13}$. Apesar de as vísceras terem alto teor de gordura saturada e colesterol, seu consumo não deve ser desencorajado, pois são importantes fontes de ferro, nutriente essencial para prevenir a anemia ferropriva, especialmente em crianças, jovens, idosos e mulheres em idade fértil'.

O grupo de carnes brancas, representado por peixes e frango, apresentou uma prevalência de consumo de $60,0 \%$ pelo menos uma vez por semana. Ortiz-Hernández e Gómez-Tello ${ }^{15}$ mostraram que $54,0 \%$ dos jovens mexicanos referiram consumir alguma vez na semana esse tipo de car$n \mathrm{e}^{15}$. O consumo de carnes brancas é recomendado devido ao baixo teor de gordura saturada que apresentam, e, no caso dos peixes, pelo seu alto conteúdo de ômega-3. Peixes de água doce e salgada são abundantes no Brasil, o que favorece o consumo de grande variedade de espécies. Entretanto, apesar da disponibilidade, o consumo de peixes foi baixo entre os adolescentes avaliados $(72,4 \%$ consomem raramente ou nunca). Confirma-se, a partir disso, que a maior parte da frequência de consumo de carnes brancas foi decorrente da ingestão de frango e não de peixes.

Constatou-se que as frequências de consumo diário dos diferentes tipos de carnes, exceto as vermelhas, foram mais elevadas entre os meninos do que entre as meninas. A POF 2008-2009 constatou um consumo diário de quase todos os tipos de carnes maior entre os meninos, com exceção das vísceras, que teve maior consumo entre as meninas ${ }^{13}$. O consumo de embutidos e carnes vermelhas foi maior entre adolescentes de melhor nível socioeconômico e entre aqueles cujas mães apresentavam maior escolaridade. Entre os adolescentes mais pobres e filhos de mães com pouca escolaridade, observou-se maior consumo de carnes brancas, principalmente carne de frango. Tal achado pode ser justificado pela praticidade e relação entre custo e consumo desses alimentos. 
Conforme já observado na literatura, o presente estudo demonstrou que quanto mais frequente o consumo de carnes, maior a prevalência de dieta rica em gordura. No presente estudo, a maior proporção de dieta rica em gordura foi observada entre os adolescentes que consumiam com maior frequência carnes vermelhas e embutidos quando comparados àqueles que faziam maior consumo de carnes brancas: fato relacionado ao alto teor de gordura saturada e colesterol presente, principalmente, nos embutidos.

\section{O N CLUS Ã O}

O estudo mostrou maiores prevalências de consumo de carnes vermelhas e embutidos e baixo consumo de peixes e vísceras entre os adolescentes avaliados. Além disso, a frequência de consumo dos diferentes tipos de carne variou conforme a condição socioeconômica dos jovens, e a frequência de consumo de uma dieta rica em gordura variou de acordo com o tipo de carne consumida.

Sendo assim, políticas podem ser pensadas com o intuito de esclarecer a composição química dos diferentes tipos de carne, possibilitando escolhas mais saudáveis além da elaboração de estratégias que estimulem o conhecimento da população acerca dos alimentos consumidos. Além disso, nossos achados reforçam a necessidade da implementação de programas de educação nutricional tanto na mídia como no ambiente escolar e podem também ser utilizados como estudo de linha de base para que se possa avaliar a tendência temporal de consumo desses alimentos.

\section{A GRADECIMENTOS}

Este artigo é baseado nos dados do estudo "Coorte de nascimentos de 1993 de Pelotas", realizado pelo Programa de Pós-Graduação em Epidemiologia, da Universidade Federal de Pelotas. A visita aos 15 anos foi financiada pela Wellcome Trust Initiative (Processo 072403///03/Z).

\section{COLABORADORES}

MCF ASSUNÇÃO, AMB MENEZES e CL ARAÚJO coordenaram o trabalho de campo, orientaram todas as etapas do trabalho e revisaram a versão final do manuscrito. SW MADRUGA e SC DUMITH auxiliaram na análise dos dados e redação do manuscrito. BC SCHNEIDER, CA VIANNA, EC MACHADO, FC WEHRMEISTER, LC MUNIZ, RV ZANINI e SP ORLANDI realizaram a análise dos dados e a escrita do manuscrito.

\section{REFERÊ NCIAS}

1. Brasil. Ministério da Saúde. Guia alimentar para a população brasileira: promovendo a alimentação saudável. Brasília: Coordenação-Geral da Política de Alimentação e Nutrição; 2006.

2. Brasil. Ministério da Saúde. Vigitel Brasil 2008: vigilância de fatores de risco e proteção para doenças crônicas por inquérito telefônico. Brasília: Secretaria de Vigilância em Saúde; 2009.

3. Kris-Etherton P, Daniels SR, Eckel RH, Engler $M$, Howard BV, Krauss RM, et al. AHA Scientific statement: summary of the Scientific Conference on Dietary Fatty Acids and Cardiovascular Health. J Nutr. 2001; 131(4):1322-6.

4. Mann Jl. Diet and risk of coronary heart disease and type 2 diabetes. Lancet. 2002; 360(9335): 783-9.

5. Mikkila V, Rasanen L, Raitakari OT, Pietinen P, Viikari J. Longitudinal changes in diet from childhood into adulthood with respect to risk of cardiovascular diseases: the cardiovascular risk in young finns study. Eur J Clin Nutr. 2004; 58(7):1038-45.

6. Ness AR, Maynard M, Frankel S, Smith GD, Frobisher C, Leary SD, et al. Diet in childhood and adult cardiovascular and all cause mortality: the Boyd Orr cohort. Heart. 2005; 91(7):894-8.

7. Sichieri R, Everhart JE. Validity of a Brazilian food frequency questionnaire against dietary recalls and estimated energy intake. Nutr Res. 1998; 18(10): 1649-59.

8. Thompson FE, Byers T. Dietary assessment resource manual. J Nutr. 1994; 124(11 Suppl):2245S-317S.

9. Onis M, Onyango AW, Borghi E, Siyam A, Nishida C, Siekmann J. Development of a WHO growth reference for school-aged children and adolescents. Bull World Health Organ. 2007; 85(9):660-7.

10. Victora CG, Araujo CL, Menezes AM, Hallal PC, Vieira MF, Neutzling MB, et al. Methodological 
aspects of the 1993 Pelotas (Brazil) birth cohort study. Rev Saúde Pública. 2006; 40(1):39-46.

11. Madruga SW, Araújo CL, Bertoldi AD. Frequency of fi ber-rich food intake and associated factors in a Southern Brazilian population. Cad Saúde Pública. 2009; 25(10):2249-59.

12. Neutzling $M B$, Araújo $C L$, Vieira MF, Hallal PC, Menezes AMB, Victora CG. Intake of fat and fiberrich foods according to socioeconomic status: the 11-year follow-up of the 1993 Pelotas (Brazil) birth cohort study. Cad Saúde Pública. 2010; 26(10): 1904-11.

13. Instituto Brasileiro de Geografia e Estatística. Pesquisa de orçamentos familiares 2008-2009: antropometria e estado nutricional de crianças, adolescentes e adultos no Brasil. Rio de Janeiro: IBGE; 2010 [acesso 2012 mar]. Disponível em: <http://www.ibge.gov.br/home/estatistica/ populacao/condicaodevida/pof/2008_2009_encaa/ default.shtm>

14. Dalla-Costa MC, Cordoni Júnior L, Matsuo T. Hábito alimentar de escolares adolescentes de um município do oeste do Paraná. Rev Nutr. 2007; 20(5):461-71. doi: 10.1590/\$1415-527320070005 00002.

15. Ortiz-Hernández L, Gómez-Tello BL. Food consumption in Mexican adolescents. Rev Panam Salud Pública. 2008; 24(2):127-35.

16. Silva ARV, Damasceno MMC, Marinho NBP, Almeida LS, Araújo MFM, Almeida PC, et al. Hábitos alimentares de adolescentes de escolas públicas de Fortaleza, CE, Brasil. Rev Bras Enferm. 2009; 62(1):18-24.

Recebido em: 14/7/2011

Versão final em: 4/5/2012 Aprovado em: 5/6/2012 